\title{
Hybrid Positron emission tomography/magnetic resonance imaging in viability assessment
}

\author{
Jeroen J. Bax, MD, PhD, ${ }^{\mathrm{a}}$ Arnold C. T. Ng, MD, PhD, , , , d and Victoria Delgado, \\ $M D, P h D^{a}$ \\ a Department of Cardiology, Leiden University Medical Center, Leiden, The Netherlands \\ b Department of Cardiology, Princess Alexandra Hospital, The University of Queensland, \\ Brisbane, QLD, Australia \\ c Centre for Advanced Imaging, The University of Queensland, Brisbane, QLD, Australia \\ d Faculty of Medicine, South Western Sydney Clinical School, The University of New South \\ Wales, Sydney, Australia
}

Received Jul 9, 2020; accepted Jul 9, 2020

doi: $10.1007 / \mathrm{s} 12350-020-02289-9$

\section{See related article, pp. 2335-2342}

In the current issue of the Journal, Kiko et al. ${ }^{1}$ have used a hybrid scanner with positron emission tomography (PET) and magnetic resonance imaging (MRI) to evaluate the presence of myocardial viability in patients with left ventricular dysfunction secondary to a chronic total occlusion of a coronary artery. The patients had moderately reduced left ventricular ejection fraction (LVEF $43.0 \pm 15.1 \%$ ), while $60 \%$ of the 255 myocardial segments were dysfunctional.

Moreover, based on tissue characterization (viability assessment), the authors aimed to predict recovery of function after percutaneous coronary intervention. PET and MRI are the "high-end imaging tools" that one can use in cardiovascular disease and they provide a wealth of information. In this specific study, the authors used resting MRI to assess the function before and (6 months) after revascularization to define functional recovery (contractile improvement) as the "gold standard" for viability. In order to characterize the tissue before revascularization (and predict recovery after revascularization), the authors used MRI with delayed contrast enhancement, which permits the delineation of dead myocardium (scar tissue), with very high resolution, and

Reprint requests: Jeroen J. Bax, MD, PhD, Department of Cardiology, Leiden University Medical Center, Leiden, The Netherlands; j.j.bax@lumc.nl

J Nucl Cardiol 2021;28:2343-5.

$1071-3581 / \$ 34.00$

Copyright (C) 2020 American Society of Nuclear Cardiology. the highest accuracy to predict no recovery after revascularization. To further characterize the dysfunctional myocardium, Kiko et al. ${ }^{1}$ have used PET with F18-fluorodeoxyglucose (FDG), which permits the assessment of residual glucose metabolism (and thus alive myocardium) and probably has the highest sensitivity to predict functional recovery.

Some issues in this study deserve attention. First, the use of hybrid PET/MRI scanners for assessment of viability is an important step forward. In the past, comparative imaging with (delayed enhancement) MRI and PET has been performed, ${ }^{2}$ but this was always based on separate MRI and PET scanners, potentially introducing misalignment of myocardial regions. With the introduction of hybrid PET/MRI scanners, co-registration will be significantly improved, resulting in increased diagnostic accuracy.

Next, resting MRI was used to assess left ventricular function before and 6 months after revascularization. Most previous studies relied on echocardiography to assess left ventricular function before and after revascularization, and although the MRI images before and after revascularization were interpreted visually, MRI clearly provides superior resolution, resulting in increased accuracy of wall motion assessment (the standard of viability assessment in the current study). Kiko et al. ${ }^{1}$ have used a 5-point scoring system, ranging from normokinesia, to hypokinesia, akinesia and dyskinesia, thereby further improving diagnostic accuracy of assessing functional recovery after revascularization. One note of caution, hypokinesia was divided in mild-moderate versus severe hypokinesia which may be challenging at times when visual scoring is used. And more specifically, 
present in 106 segments $(69.7 \%)$, whereas both imaging techniques showed non-viability in $11.2 \%$. This resulted in an agreement of $80.9 \%$ of both techniques for viability/scar tissue. The disagreement between the 2 techniques may be related to the differences in resolution, and the inherent differences between MRI providing (anatomical) scar imaging, and PET providing (metabolic) functional imaging. Moreover, scar segments on PET and MRI had significantly worse wall motion score at baseline $(1.60 \pm 0.71$ and $1.56 \pm 0.68$, respectively) as compared to viable segments $(1.90 \pm 0.77$ and $2.00 \pm 0.77$, respectively). At 6 months follow-up (after revascularization), 94 of the $152(61.4 \%)$ dysfunctional segments improved in function (which is a relatively high percentage, but may be related to development of collaterals). Importantly, recovery of function occurred in $67.8 \%$ of the PET viable segments and in $71.5 \%$ of the MRI viable segments. Conversely, recovery of function occurred only in $23.5 \%$ and $20.7 \%$ non-viable segments on PET and MRI, respectively. However, significant improvement in diagnostic accuracy occurred with the hybrid PET/MRI scanner: $77.4 \%$ of the viable segments improved in function and only $11.8 \%$ in the non-viable segments. These results highlight the use of hybrid PET/MRI scanners for future viability assessment. The strength of the PET/MRI scanners relates to the integrated, highresolution "anatomical" assessment of tissue with MRI (ventricular scar assessment) and high-resolution "functional" assessment of tissue with PET (ventricular glucose utilization). This improved (integrated) tissue characterization will not only improve accuracy in viability/scar assessment, but also contribute to improved assessment of systemic diseases with cardiac involvement (e.g., sarcoidosis, myocarditis, and amyloidosis).

\section{References}

1. Kiko T, Yokokawa T, Misaka T, Masuda A, Yoshihisa A, Yamaki $\mathrm{T}$ et al (2020) Myocardial viability with chronic total occlusion assessed by hybrid Positron emission tomography/magnetic resonance imaging. J Nucl Cardiol. https://doi.org/10.1007/s12350-02002041-3

2. Klein C, Nekolla SG, Bengel FM, Momose M, Sammer A, Haas F et al (2002) Assessment of myocardial viability with contrastenhanced magnetic resonance imaging: Comparison with Positron emission tomography. Circulation 105:162-167

3. Tillisch J, Brunken R, Marshall R, Schwaiger M, Mandelkern M, Phelps $M$ et al (1986) Reversibility of cardiac wall-motion abnormalities predicted by positron tomography. N Engl J Med 314:884-888

4. Baer FM, Voth E, Deutsch HJ, Schneider CA, Horst M, de Vivie ER et al (1996) Predictive value of low dose dobutamine transesophageal echocardiography and fluorine-18 fluorodeoxyglucose positron emission tomography for recovery of regional left reasing transmurality of scar tissue on contrastenhanced MRI. More specifically, of the 152 dysfunctional segments, viability on both PET and MRI was 
ventricular function after successful revascularization. J Am Coll Cardiol 28:60-69

5. Bax JJ, Visser FC, Elhendy A, Poldermans D, Cornel JH, van Lingen A, Boersma E, Sloof GW, Fioretti PM, Visser CA (1999) Prediction of improvement of regional left ventricular function after revascularization using different perfusion-metabolism criteria. J Nucl Med 40:1866-1873

Publisher's Note Springer Nature remains neutral with regard to jurisdictional claims in published maps and institutional affiliations. 